\title{
Electrical characterization of microdischarges produced by dielectric barrier discharge in dry air at atmospheric pressure
}

\author{
N. Jidenko ${ }^{1}$, M. Petit ${ }^{2}$ and J.-P. Borra ${ }^{1}$ \\ ${ }^{1}$ Laboratoire de Physique des Gaz et des Plasmas (CNRS -Univ. Paris XI) \\ Equipe Décharges Electriques et Environnement, Supelec, 91190 Gif sur Yvette, France \\ ${ }^{2}$ Service Electrotechnique et Electronique Industrielle, SUPELEC Plateau de Moulon, \\ 91192 Gif sur Yvette Cedex, France \\ E-mail: nicolas.jidenko@pgp.u-psud.fr
}

\begin{abstract}
This work addresses the case of millimetre sized plane-to-plane dielectric barrier discharge with sinusoidal voltage between 1 and $60 \mathrm{kHz}$ for different configurations in air at atmospheric pressure. The first aim of this work is to achieve a representative statistical analysis of the electrical characteristics of the microdischarges. The numerical data treatment presented here, enables to determine the maximum current, the duration, the amount of charge, and the triggering voltage for each current pulse. Both the average values as well as the deviations are checked. The relative influence of the operating parameters (voltage, gas flow rate, gap width, frequency) on the local surface polarization, electron attachment and temperature affecting microdischarge characteristics has been depicted in different arrangements. A special attention is paid to the influence of dielectric walls inside the discharge gap, perpendicular to the electrodes. Besides, this study allows the identification of the operating conditions for which all the microdischarges can be considered identical to study the local physical and chemical processes around each microdischarge.
\end{abstract}

\section{Introduction}

Electrical discharges will occur between two conducting electrodes, separated by a gas layer, if the breakdown value of the electric field strength is reached (about $30 \mathrm{kV} . \mathrm{cm}^{-1}$ for air at atmospheric pressure). Dielectric barrier discharges (DBD) are characterized by the presence of at least one insulating layer (dielectric barrier) between electrodes. The dielectric barrier prevents arc formation but involves an alternative polarization of the system. Regardless of the electrodes geometry, the discharges mainly occur as numerous filaments distributed temporally and spatially. In the plane-to-plane arrangement, the Laplace field (field without spatial or surface charge) is homogeneous; however the discharge is characterized by the Poisson field (taking into account volume and surface charges). The filamentary discharges are termed "microdischarges" as they only last a few tens of nanoseconds and have a diameter of 10-100 $\mu \mathrm{m}$ [1-5].

The cylindrical volume around a microdischarge in which no subsequent microdischarge occurs until the next half period, in our conditions (maximal peak-to peak voltage of $20 \mathrm{kV}$ ), will be referred here after as the "electrostatic influence volume" of a microdischarge. The microdischarge lays charges on the dielectric surface which reduce the electric field in the influence volume to below the extinction field strength until the next period. During the next period, the charges deposited on the surface increase the field strength until the development of a new microdischarge at the same location can occur. For a given geometry, the number of microdischarges per half period, distributed in time and space on the total surface of the plane-to-plane DBD, depends almost entirely on the applied voltage with the frequency of the voltage playing only a minor role [1]. On first inspection, microdischarges occur at the same location, thus the evolution of the electric field profile is repeated at the frequency of the applied voltage. The plane-toplane DBD enables the reproduction of similar microdischarges with a controlled number per unit time and area with respect to the voltage and frequency. The mean energy per microdischarge can only be varied by changing the gap width and dielectric specific capacitance (ratio between dielectric permittivity and dielectric thickness).

DBD are utilised in various applications such as ozone production, pollution control by oxidation of volatile organic compounds or nitrogen monoxide, bio-treatment of micro-organisms by oxidation, surface treatment (thin film deposition, wettability modification), UV or VUV generation, aerosol charging and electro-filtration [4-8]. 
In all these applications, for a given reactor at a constant flow rate, the energy per microdischarge and the number of microdischarges per unit time and area are the key parameters for process optimisation. Actually, the unitary production rates of reactive species per microdischarge depend on the energy per microdischarge and on the coupling of the microdischarges (number of microdischarge per unit time and area) controlling the temperature and the densities profiles in the reactor. There are a number of different models which describe the development of a single filament based on the electron energy distribution (related to discharge energy) $[9,10]$. Models usually assume that plane-to-plane discharge is composed of a uniformly spaced array of identical axi-symmetric microdischarges [3,9]. This approximation is valid but improved knowledge of the distributions of the electrical characteristics (maximum pulse current, duration at mi-height, pulse charge, and threshold voltage cf. below) could improve the dielectric barrier discharges models and should aid process optimisation. Moreover, experimental study of the local physical and chemical processes occurring around each microdischarge can be driven through macroscopic measurements if microdischarges can be considered all identical. Thus, the identification of the operating conditions in which all the microdischarges are identical is of a major interest.

Some studies deal with the statistical distribution of the electrical characteristics of microdischarges. Kreuger et al. developed a statistical characterization of the phase of partial discharges with a digital recording system [11]. Drimal et al used a pulse amplitude analyzer to statistically study the amount of charge by microdischarge [12]. Gibalov et al. also studied the distribution of the transferred charge value by digitalizing of the current [13].

Whatever the application, the processes (production, transport, kinetics in volume and on surface), the variables on which they depend (density, temperatures and their gradients) and their evolutions are related with the characteristics of plasma discharges (energy per microdischarge and space-time distribution of the microdischarges). The characteristics of the discharges are controlled by the operating parameters: voltage, frequency, gap width, nature of materials of the reactor and nature, and operating conditions (gas flow rate, temperature and moisture).

A complete method for the measurement of main unitary electrical characteristics of the microdischarges and their distributions has been developed. The characteristics of the pulse are:

The maximum of the pulse current $\left(I_{0}, \mathrm{~mA}\right)$ is reached once the channel formed just after the streamer strikes the cathode. It can be used to account for the plasma surface interaction related to the heating of the surface spot through thermal effects and also the energy of ions impinging on the surface.

The amount of charges involved in the microdischarge $(Q, \mathrm{nC})$, is related to the number of chemical species created and to the temperature of neutral species in the plasma.

The duration ( $\Delta t, \mathrm{~ns})$ is measured at $50 \%$ of the maximum to avoid considering as one single microdischarge a pulse composed of several microdischarges and not to perturb the measurement with oscillations of the current. The duration is composed of two parts. The rise time of the current (approximately $1 \mathrm{~ns}$ ) and mainly the decay of the current related to the electron attachment phase due to the reduction of the reduced field in the microdischarge.

The instantaneous value of the applied voltage when the pulse occurs $\left(V_{s}, \mathrm{kV}\right)$ allows the evaluation of the influence of the charge deposited on the dielectric surface.

The method includes an adjustment of the electrode surface to limit the number simultaneous microdischarges and is based on a numerical treatment of oscilloscope measurements, excluding multiple pulses. The data treatment allows the evaluation of the unitary electrical characteristics, the total number of microdischarges and the calculation of a macroscopic current.

Different discharge arrangements are compared through the study of macroscopic characteristics integrated over the whole reactor (input power or currents) and the electrical characteristics of the microdischarge. In a wire-to-wire arrangement, the microdischarges are only distributed in one dimension allowing validation of the pulse treatment. The influence of the operating parameters (voltage, gas flow rate, temperature, gap width, frequency) on the electrical characteristics of the microdischarge was established using different discharge arrangements to deal with the distribution of the microdischarges in time and space. 


\section{Material and methods}

\subsection{Experimental set up}

The experimental arrangement is shown in Figure 1. Three different sinusoidal high voltage generators were used. The frequency was able to be adjusted from 1 to $60 \mathrm{kHz}$ and with a maximum peak-to-peak voltage of $20 \mathrm{kV}$. This AC high voltage $u(t)$ is applied across the cell electrodes. The four different cells used are described below.

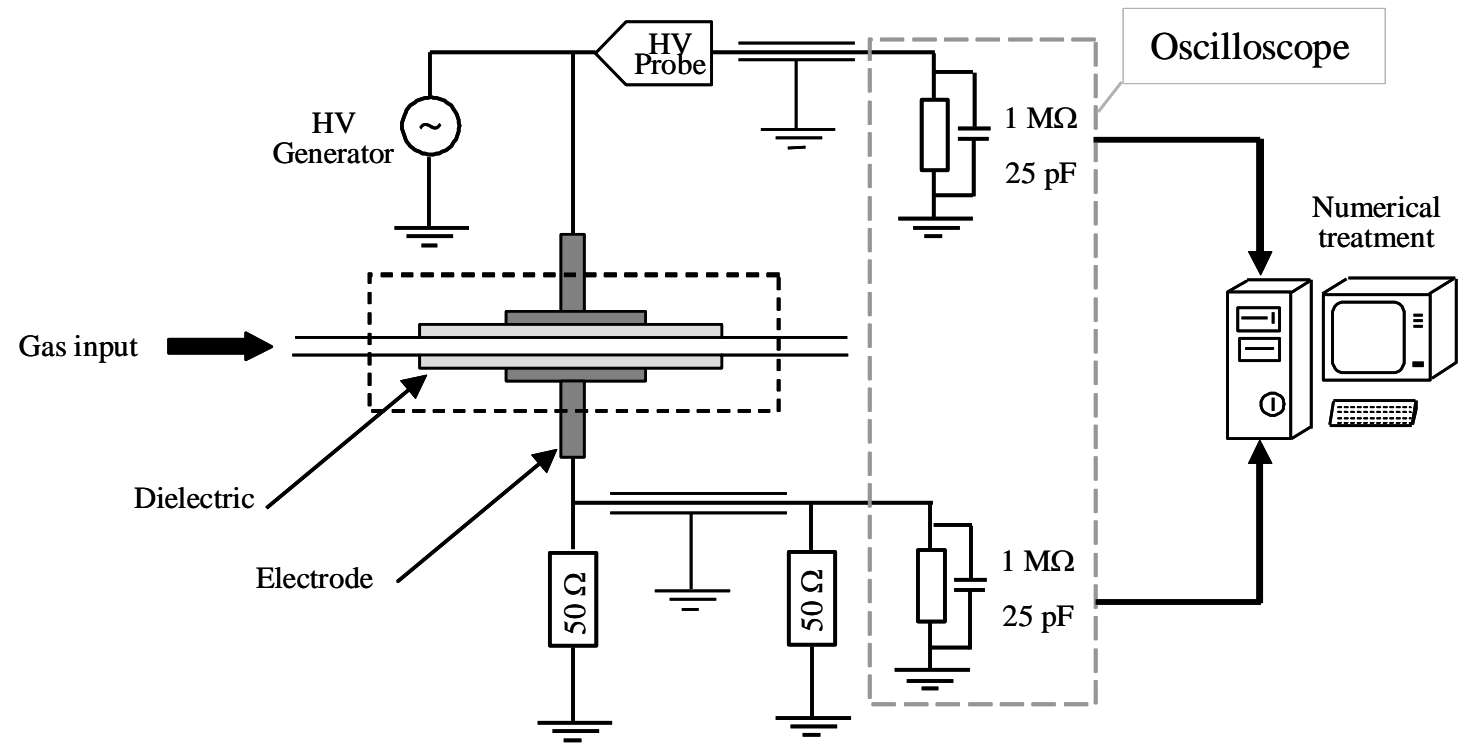

Figure 1: Experimental set-up.

The flow rate of dry air (Relative humidity $<5 \%$ ) is controlled with mass flow controllers between 0.2 and $24 \mathrm{~L} \mathrm{~min}^{-1}$. Thus, the maximum mean gas velocity (gap width $1 \mathrm{~mm}$ ) is $40 \mathrm{~m} \mathrm{~s}^{-1}$ and the residence time lies between 120 and $2 \mu$ s.

\subsection{Description of the discharge cells}

Four kinds of double dielectric barrier discharge are compared in this study. The dielectrics are alumina and cordierite. Three cells were made with two parallel alumina plates (thickness e $=0.55 \mathrm{~mm}, \varepsilon_{\mathrm{r}} \sim 9$ ). One cell had electrodes of $1 \times 3 \mathrm{~cm}^{2}$ area, with a $1 \mathrm{~mm}$ gap width, while the second had the same electrode area but a gap width of $0.5 \mathrm{~mm}$. In the third cell, the electrodes are two stainless wires (diameter: $100 \mu \mathrm{m}$ and 1 $\mathrm{cm}$ long) which are perpendicular to the flow; the gap width between the alumina plates measures $1 \mathrm{~mm}$.

The last cell was a single stage of a cordierite monolith $\left(\varepsilon_{\mathrm{r}} \sim 5\right)$ (used in pollution control reactors cf. Figure 2). The area of the electrodes is $1 \times 3 \mathrm{~cm}^{2}$. The square channels of $1 \mathrm{~mm}$ impose the discharge gap. The width of the electrodes $(1 \mathrm{~cm})$ corresponds to 9 pores. In this arrangement, the walls of the dielectrics (perpendicular to the electrodes) lead the microdischarges coupling volume and surface discharges.

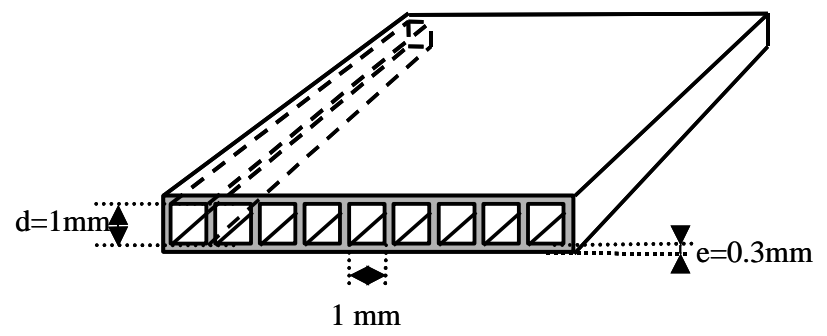

Figure 2: The cordierite dielectric geometry.

For all the cells, a silicon paste surrounding the electrodes prevents arcs and other parasitic discharges occurring outside of the gap. The cells are conditioned between two half casings made of stumatite (aluminosilicate). The electrodes are designed to minimize edge effects. 


\subsection{Diagnostics}

\subsubsection{Electrical measurements}

The AC high voltage was measured with a high voltage probe (Tektronics 6015A Bandwidth $75 \mathrm{MHz}$ ). The current in the circuit was measured through a $50 \Omega$ adaptive line to prevent deformation and reflection occurring. This is equivalent to a $25 \Omega$ resistor.

- Relation between charge displacements in the gap and the measured current The study of the current induced by the discharges can be achieved thanks to the conservation of energy. The electric energy injected in the cell is the sum of the energy transferred to the gas via the collision of the electrons and of the variation of the electrostatic energy stored in the cell,

$$
u(t) \cdot i(t)=\int_{V}^{\rho} j(t) \cdot \hat{E}(t) \cdot d v+\int_{V} \varepsilon \cdot \frac{\partial \tilde{E}}{\partial t} \cdot \hat{E}(t) \cdot d v
$$

where $u$ is the applied voltage, $j$ is the local flux of charge $\left(A \cdot \mathrm{m}^{-2}\right), E$ is the electric field $\left(\mathrm{V} \cdot \mathrm{m}^{-1}\right)$ and $\int_{\mathrm{V}} \mathrm{dv}$ is a volume integral over the volume between the electrodes including the dielectrics.

The movement of each charge in the gap affects the measured current. Sato $[14,15]$ demonstrated that with metallic electrodes, the current in the external circuit induced by the displacements of charge in the gap $\left(i_{\text {ind }}\right)$ is,

$$
i_{\text {ind }}(t)=\frac{1}{u(t)} \int_{V}^{\rho} j(t) \cdot E_{L}(t) \cdot d v
$$

where $E_{L}$ is the Laplace field (field without space and surface charge).

Moreover, the dielectric properties (the thickness of the dielectrics $e$ and the relative permittivity of the dielectric $\varepsilon_{r}$ ) affect the influence of a charge movement in the gap on the current in the external circuit. Raether gives the relation between the charge left on surface $\left(Q_{r}\right)$ and charge on the electrodes $\left(Q_{m}\right)$ [16].

$$
\frac{Q_{r}}{Q_{m}}=1+\frac{C_{g}}{C_{d}}=1+\frac{2 . e}{\varepsilon_{r} d}=\alpha
$$

where $C_{g}$ is the capacitance of the gas gap; $C_{d}$, the capacitance of the dielectrics; and $d$, the gap width between the two dielectrics. Here the dielectric thickness e varies between $300 \mu \mathrm{m}$ (cordierite) and 550 $\mu \mathrm{m}$ (alumina) but the silicon paste has to be taken into account, so that $Q_{m}$ represents between $60 \%$ and 95\% of $Q_{r}$ for the plane-to-plane arrangements. Drimal et al. have determined the ratio $Q_{r} / Q_{m}$. The experimental values correspond to only about half of the theoretical values calculated with the relation (1) [17]. The difference between the theoretical curve and experimental results may reveal the difference between a 3D-process and 1D-model of this process according to one of the author. Moreover, the displacements of non collected ions remaining in the gap after the microdischarge (because of surface polarization) probably account for a part of the difference.

\section{- Calculation of the instantaneous discharge current}

Because of the silicone paste between electrode and dielectric, $C_{d}$ and $\alpha$ can not be calculated. The Lissajous method could be used to evaluate these parameters [18]. However, equation (3) also describes the ratio between the applied voltage and the potential difference between each side of the gap with an homogeneous field (without discharge nor charge deposited on surface). In this work, the threshold voltage $\left(V_{s}\right)$ corresponds to the voltage of the first microdischarge, without charge deposited on dielectrics. $V_{s}$ allows the evaluation of this ratio $\alpha$. 
The current $i(\mathrm{I})$ measured in the external circuit contains two main components: a capacitive current $i_{c}(t)$ or displacement current which flows with a time-dependent applied voltage and the discharge current $i_{d i s}(t)$. Using a digital storage oscilloscope (LeCroy LC374A, $500 \mathrm{Mhz}, 2 \mathrm{Gs} / \mathrm{s}$ ) and MATLAB ${ }^{\circledR}$, both current and voltage are treated as described in previous paper [19]. The oscilloscope memory (up to 250 kpts) and its segmentation (up to 500 segments) allow pulses to be recorded with a high sampling rate (up to $0.5 \mathrm{~ns} / \mathrm{sample}$ ).

The capacitance (C) of the cells is evaluated, without discharge, from the ratio between current and voltage amplitude divided by 2.П.F. Then, for each voltage, the capacitive current (calculated from the voltage signal and the capacitance) is withdrawn from the measured current, allowing the analysis of the current induced by the discharge $i_{\text {ind }}$ (with $i_{\text {ind }}=i-i_{c}$ ).

Assuming that the equation (3) is still valid, the discharge current of discharge is thus calculated according to,

$$
i_{\text {dis }}(t)=\alpha \cdot i_{\text {ind }}=\left(1+\frac{C_{g}}{C_{d}}\right) \cdot\left(i(t)-C \frac{d u(t)}{d t}\right)
$$

where $C$ is the capacitance of the cell ( $C_{d}$ and $C_{g}$ in series), as described by Bibinov [20].

- Limitation of number of the series using a small electrode for the measurement

Each microdischarge induces a current pulse (about $20 \mathrm{~ns}$ ) in the external circuit. It follows that if the electrode is a solid plane, some of the induced pulses merge. It is rather difficult to distinguish the contribution of each single microdischarge among the others on the resulting current. Series of microdischarges [18] and chains of microdischarges [20] are the terms used to describe the fact that several microdischarges are triggered, at different positions in the gap, in a time lapse smaller than the duration of a microdischarge or even at the same time. Murata et al. proved that the series occur in limited space of about one square centimetre. They proposed an explanation for the occurrence of a group of microdischarges. The UV emission from a preceding microdischarge can preionize the air and triggers the temporal superposition of microdischarges [22].

The grounded electrode was thus divided into two parts to limit the number of series per period and the number of pulse per series. A circular electrode $(8 \mathrm{~mm}$ diameter to collect several microdischarges per period) was inserted in the middle of the drilled electrode and separated by a thin Teflon tube for insulation. Each part was connected to the oscilloscope to record the corresponding induced current pulses. Even with the small electrode, microdischarge can occur at the same time (cf. section 3.2.2).

\subsubsection{Numerical treatment}

Previous work describes the numerical treatment [19], which was originally developed for point-to-plane dielectric arrangement but has been since adapted to plane-to-plane [23]. The aim is to acquire data on the number and the shape of the current pulses. The pulses have to be separated from the capacitive current: each current pulse or each series of current pulses is separated.

First of all, the acquired current is corrected with equation (1). Each positive (negative) pulse is detected from its maximum (minimum). From slope considerations the beginning and the end of the pulse can be determined. The corrected current signal, rid of the pulse (single microdischarge or series), is obtained by replacing the pulse by a straight line between the beginning and end. This current is the sum of capacitive and non-impulsive current. The non-impulsive current is obtained by subtracting the capacitive current. This current should be null if the current is only capacitive and impulsive.

Each pulse is stored to construct the impulsive current. Meanwhile, the height, the width at half maximum, the charge, and the onset voltage are stored for each pulse.

The segmentation of the scope memory allows the acquisition of positive pulses with a high sampling rate and even more in the case of a single microdischarge per half period (up to 500 pulses can be stored). 


\subsubsection{Drawbacks and solutions}

Even with an adapted line, some oscillations of current can occur because of parasitic inductances of high voltage and earth cables. Using short cables can reduce oscillations.

As the grounded electrode is divided into two parts (cf. section 2.3.1), microdischarge influences both parts. Nevertheless, a dominant contribution on the current is obtained on the part that is under the microdischarge. Only dominant pulses are taken into account; both the duration of the pulse and the height allow the identification of the different kind of pulses.

The procedure required an oscilloscope with a high sampling rate $(0.5 \mathrm{~ns} / \mathrm{sample}$ or even less $)$ and a wide memory for a maximum resolution over several periods. For frequencies below $10 \mathrm{kHz}$, the memory of the scope used is too weak. Thus, only scope memory segmentation can allow the statistical study of the pulses.

The numerical treatment highlights a non-impulsive current (cf. section 2.3.2) different from the capacitive one as already presented in [24]. This current represents between 0 and $20 \%$ of the instantaneous current.

Four hypotheses can be devised to account for this non-impulsive current:

- This current can be induced by losses either in the dielectrics or by conduction on the surface of the ceramic casing. In that case, the non-impulsive current is proportional to the voltage. This hypothesis can be excluded for two reasons. The current measurement without discharge proves that losses are negligible. Moreover, the non-impulsive current is in phase with the "pulses-period" and does not exist in the dead time without pulse.

- Independent electron avalanches leading to insufficient electron density to account for a full streamer development can be involved in this current.

- This current can be induced by the migration of charge remaining in the gap either in the volume or on the surface after microdischarge development when surface potential repels the oncoming last positive ions produced by the primary streamer.

- Lastly, the pulse end is difficult to detect and a part of charge pulse can be excluded from the pulse. But it represents less than a few percent of the pulse charge.

However, this non-impulsive current represents, in the worst case, less than $20 \%$ of the current. In the following, this current is not taken into account to deal with microdischarge. The discharge current is considered as being only impulsive.

\section{Experimental results}

\subsection{Comparison of the reactors according to macroscopic currents}

The aims of this paragraph are to highlight the advantage of the method (cf. section 2.3.2) on the macroscopic characterization and to distinguish the successive discharge operating modes related to the distribution of filamentary discharges in time and space. Two macroscopic currents are derived from electrical measurements. Then the current-voltage characteristics are compared for different cells to deal with distribution of the microdischarge. The active current is calculated from input power measurement, but the calculation of the total current implies the microdischarge treatment. The frequency is set to $60 \mathrm{kHz}$ (cf. section 2.3.3).

\subsubsection{Definition of macroscopic currents}

The instantaneous current has two main components: (i) a capacitive one (ii) pulses (similar in both polarities) associated to filamentary microdischarges. On the Figure 3 (a), both instantaneous voltage and current are plotted for a peak-to-peak applied voltage of $13.2 \mathrm{kV}$. A zoom of a single microdischarge is represented in Figure 3 (b) and a zoom of a series in Figure 3 (c). 

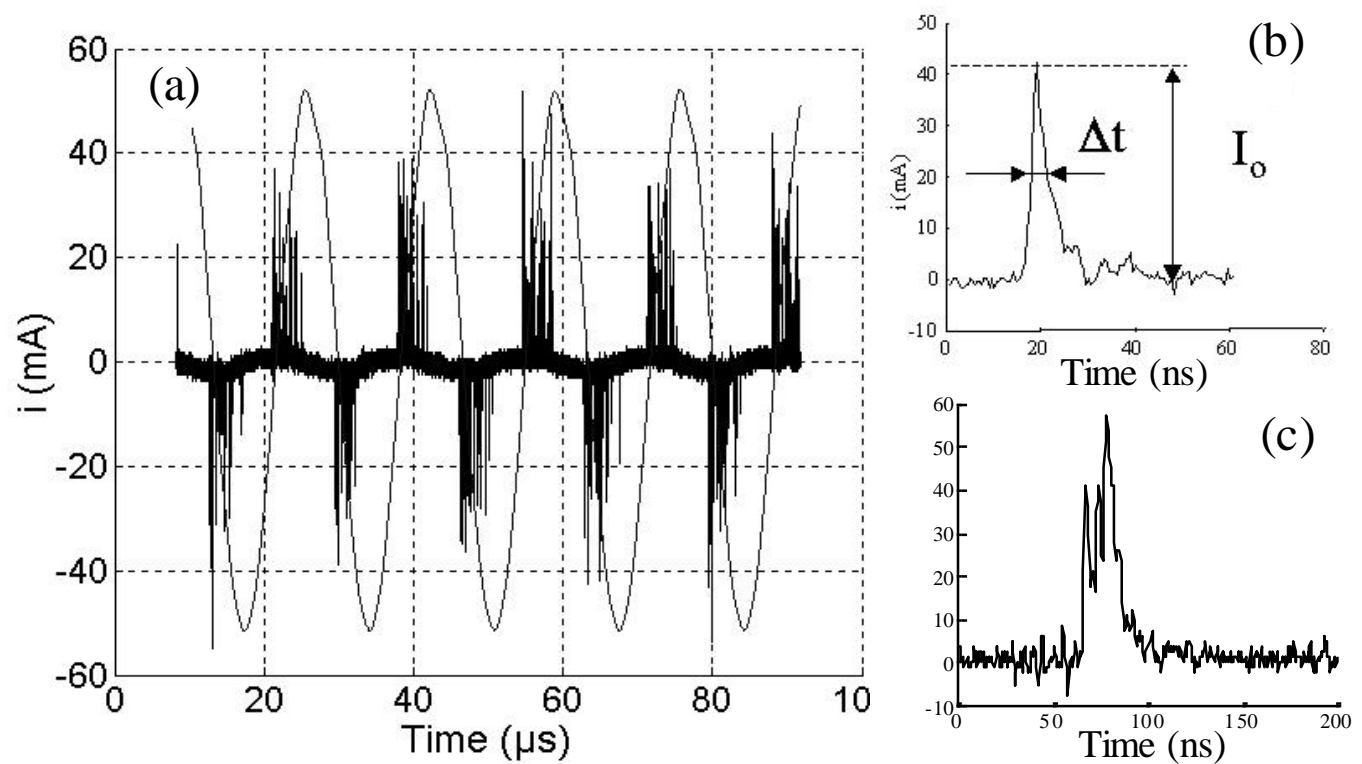

Figure 3: (a) Voltage and instantaneous current (b) single current pulse (c) series of current pulses.

For high voltage, the charges deposited on the dielectric during the last half period allow some pulses to occur before the voltage inversion (Figure 3 a). In this phase, the discharge cell "acts as a generator", from an electrical circuit point of view, due to the electrostatic energy stored in the discharge cell. Instant power is thus negative, whereas some discharges are efficient to produce active chemical species.

With DC discharges, the average current-voltage characteristic is used to identify the different discharge regimes. With DBD, the average current is null. Consequently, a macroscopic current has to be defined.

Usually, the active current $I_{\text {act }}$ defined as $P / V_{r m s}$ (where $P$ is the average power and $V_{r m s}$ is the root mean square voltage) is used [25].

$$
I_{a c t}=\frac{\frac{1}{n T} \int_{n T} u(t) \cdot i(t) \cdot d t}{\sqrt{\frac{1}{T} \int_{T} u^{2}(t) \cdot d t}}
$$

where $T$ is the period of voltage oscillation and $\mathrm{n}$ an integer.

The active current $I_{a c t}$ can be used to evaluate the average current provided by the generator to the discharge. But, it is not representative of the electrical, thermal, and chemical properties of the plasma because of the electrical losses. However for DC measurement, the number of ozone molecules per impulse charge unit is characteristic of each discharge regime [25] and to take into account all the microdischarges according to their charge in absolute value (even if they occur before the voltage inversion) the total current $I_{t o t}$ is defined as the sum of the impulsive charge per unit time (with $Q_{p}$ the mean charge per pulse, $N_{p}^{(T)}$ the number of microdischarge per period and $\mathrm{F}$ the frequency),

$$
I_{\text {tot }}=<\mathrm{Q}_{\mathrm{p}}>.<N_{p}^{(T)}>. F
$$

An approximated value of $I_{t o t}$ can be obtained by,

$$
I_{t o t} \sim \frac{1}{\Delta T} \cdot \int_{\Delta T}\left|i_{d i s}(t)\right| . d t
$$


In this study, the total current is calculated according to:

$$
I_{\text {tot }}=\frac{1}{n T} \cdot \sum_{i}\left|Q p_{i}\right|
$$

where $Q p_{i}$ the corrected charge of the $\mathrm{i}^{\text {th }}$ pulse during $n T$.

\subsubsection{Wire-to-wire arrangement}

In this arrangement, the electric field is no longer fully homogeneous; however the same kind of streamer like filamentary discharge occurs as in the plane-to-plane arrangement. Moreover, the small area of the wire implies a weak number of filaments per unit area and time and a limited number of series which allows the validation of the method used for pulse treatment. Finally, the study of physical and chemical processes around each microdischarge is simpler in this case since the gas is not spatially retreated by successive microdischarge.

The current analysis is thus easier than with a greater electrode area. The two macroscopic currents defined in 3.1.1 are plotted in Figure 4 (a) versus peak-to-peak voltage for the wire-to-wire arrangement. Figure 4 (b) represents the number of detected pulses including series (dotted line) and the total number of microdischarges (black line). The total number is obtained after treatment by splitting series in number of individual microdischarges by dividing the charge of the each series by the mean amount of charge per microdischarge.
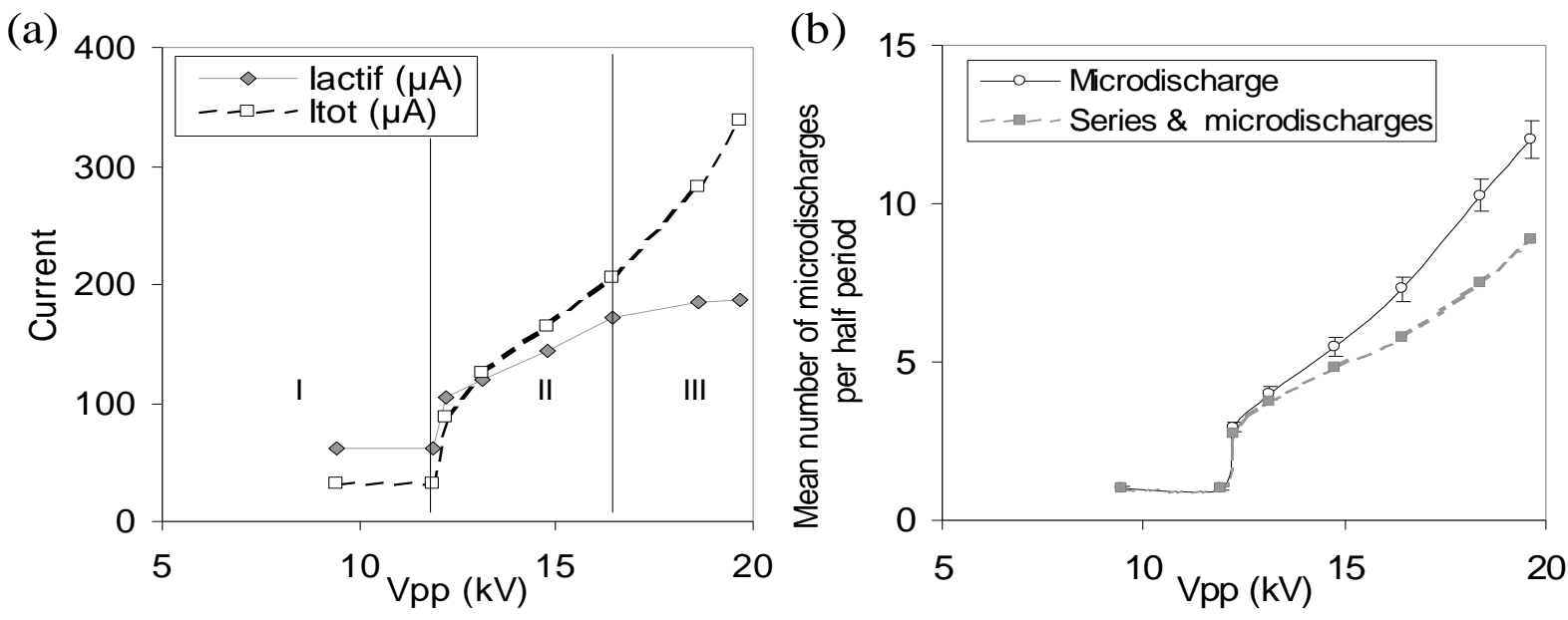

Figure 4: (a) Active and total currents (b) Number of pulses versus applied voltage for the DBD wire-to-wire.

In Figure 4 (a), the two current-voltage characteristics allow the distinction between successive discharge operating modes related to filamentary discharges spatial and temporal distribution. The three operating modes can be differentiated.

From 9 to $12 \mathrm{kV}$ (zone I), $I_{a c t}$ and $I_{\text {tot }}$ are nearly constant because only one microdischarge occurs per half period and the charge per pulse increases by only few percent. The microdischarge is located at a particular site. Either an imperfection in the dielectric or a speck on the surface can reinforce the electric field at this location. The increase of the voltage leads to a reduction of the duration of the microdischarge with a nearly constant charge per pulse probably due to a higher electric field (cf. Figure 9 and section 3.2.2.).

From 12 to $16 \mathrm{kV}$ (zones II), discharges occur along the length of the wires. The number of microdischarges (and thus the currents) rises linearly with voltage, related to the number of microdischarges. 
Above $16 \mathrm{kV}$ (zone III), the active current saturates (due to "subtraction" of pulses occurring before voltage inversion), both total and discharge currents increase. A small increase of these currents slope is observed for the number of microdischarges. The surface polarization limits the development of supplementary microdischarges in the wires axis. But a high electric field can allow the development of microdischarges on both sides of the axis, explaining the increase in microdischarge number.

In Figure 4 (b), as the mean charge per microdischarge is nearly constant (cf. Figure 9 and section 3.2.2.), the increase of the currents with voltage is thus related to the increase of the number of microdischarges per unit area and time. Even with a $1 \mathrm{~cm}$ wire long, series of microdischarges may occur as soon as two microdischarges take place in the same half period. This tends to support that the hypothesis of photoionization triggering the series of microdischarges is not sufficient to explain the occurrence of the series of microdischarges. Series can also occur without photo-ionization. The probability of two independent microdischarges occurring in less than the discharge duration is not null and depends on electric field value in the volume.

\subsubsection{Comparison of two arrangements}

The density per unit area of macroscopic parameters (input power, microdischarge number or current) allows the comparison of reactors with different electrode areas. The current density is defined as the ratio of the current on the electrode area. Moreover, the voltage operating ranges are different because the dielectric materials have different thickness and dielectric permittivity. To compare similar field condition in the gap, taking into account the specific capacitance of the dielectrics both the threshold voltage ( $V_{f}$ : the peak-to-peak voltage when the first microdischarge occurs during the "first" switch on of the reactor i.e. without any surface charge left by previous discharges) and $\alpha$ factor of each system are used (cf. section 2.3.1). Thus, the densities of the two currents corrected by $\alpha$ factor defined in 3.1.1 are plotted versus $1 / \alpha .\left(V_{p p}-V_{f}\right)$ in the two different cells (alumina: $V_{f}=16 \mathrm{kV} ; \alpha=1.60$; Figure 5 (a) and cordierite: $V_{f}=14.5 \mathrm{kV} ; \alpha=1.45$; Figure 5 (b)).
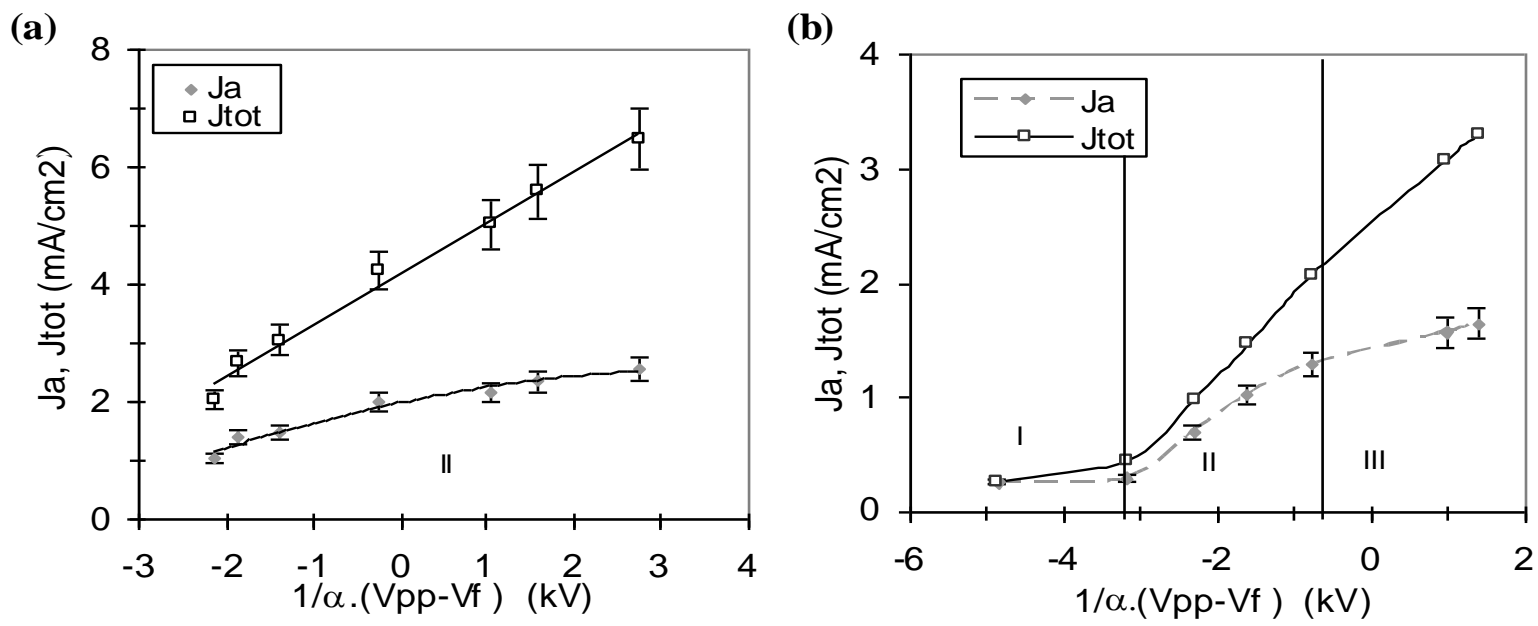

Figure 5: $J_{t o t}, J_{a}$ versus voltage for (a) alumina (b) cordierite.

For the alumina cell in Figure 5 (a), the total current increases linearly with the voltage. Zone I and III, identified with the wire-to-wire arrangement, do not appear with alumina plane-to-plane. Zone I is difficult to observe because the surface of the dielectrics is smooth (Surface roughness $R_{\max } \sim 0.08 \mu \mathrm{m}$ ) and a voltage increase of few hundred volts implies a change of the microdischarge numbers. Zone III may probably exist for higher voltage. 
For the cordierite cell (Surface roughness $R_{\max } \sim 5 \mu \mathrm{m}$ ) in Figure $5(\mathrm{~b})$, the same three operating modes exist as with the wire-to-wire arrangement.

When the current densities in the two arrangements are compared, it will be noted that higher current densities are produced with alumina for a similar over-threshold Laplace field in the gap. There are two possible explanations for this. The first is that the cordierite arrangement contains walls leading to a reduction of the free space to develop discharges; that can be corrected and accounted for by only $10 \%$. Microdischarges are smaller and more rapid in the cordierite arrangement.

The macroscopic currents allow the comparison of different discharge cells and even an initial approximation of the distribution of the microdischarges in time and space. However, the following study of the individual microdischarges characteristics gives useful information on the distribution of the microdischarges in time and space.

\subsection{Electrical characteristics of microdischarge: parametric influence}

The current of a single filamentary discharge in a plane-to-plane discharge arrangement is plotted in Figure $3 \mathrm{~b}$ ). The instantaneous current is treated as described in section 2.3.2. The four main characteristics of the current pulse are presented in the introduction. The method to study individual microdischarges is described. Then, the influences of operating parameters (voltage, gas flow rate, temperature, gap width, frequency) on the electrical characteristics of the microdischarges are presented.

\subsubsection{Validation of the protocol of exclusion of the series}

The voltage influence is characterized in the different arrangements for a given frequency of $60 \mathrm{kHz}$ and a gap width of $1 \mathrm{~mm}$. First, the use of the wire-to-wire arrangement at low voltage allows the validation of the measurement method and the identification of the series of microdischarges. In fact, the low number of microdischarge per half period reduces the number of series so that the data treatment is easier. From this arrangement, we define a treatment protocol to give the total number of microdischarges independently from their eventual simultaneity (series).

As the system is symmetric, positives and negative pulses are similar. Only the positive pulses are stored using the segmentation of the scope memory. The first distributions presented concern low voltage without influence of charge deposit on succeeding microdischarges at different locations. The distributions of the measured characteristics of the microdischarge in the wire arrangement for 11 (black) and $12 \mathrm{kV}$ (gray) are plotted without any correction in Figure 6 . At $11 \mathrm{kV}$, only one microdischarge occurs per half period, 500 pulses are treated. At $12 \mathrm{kV}$, two or three microdischarges occur per half period and 1300 pulses are treated. 

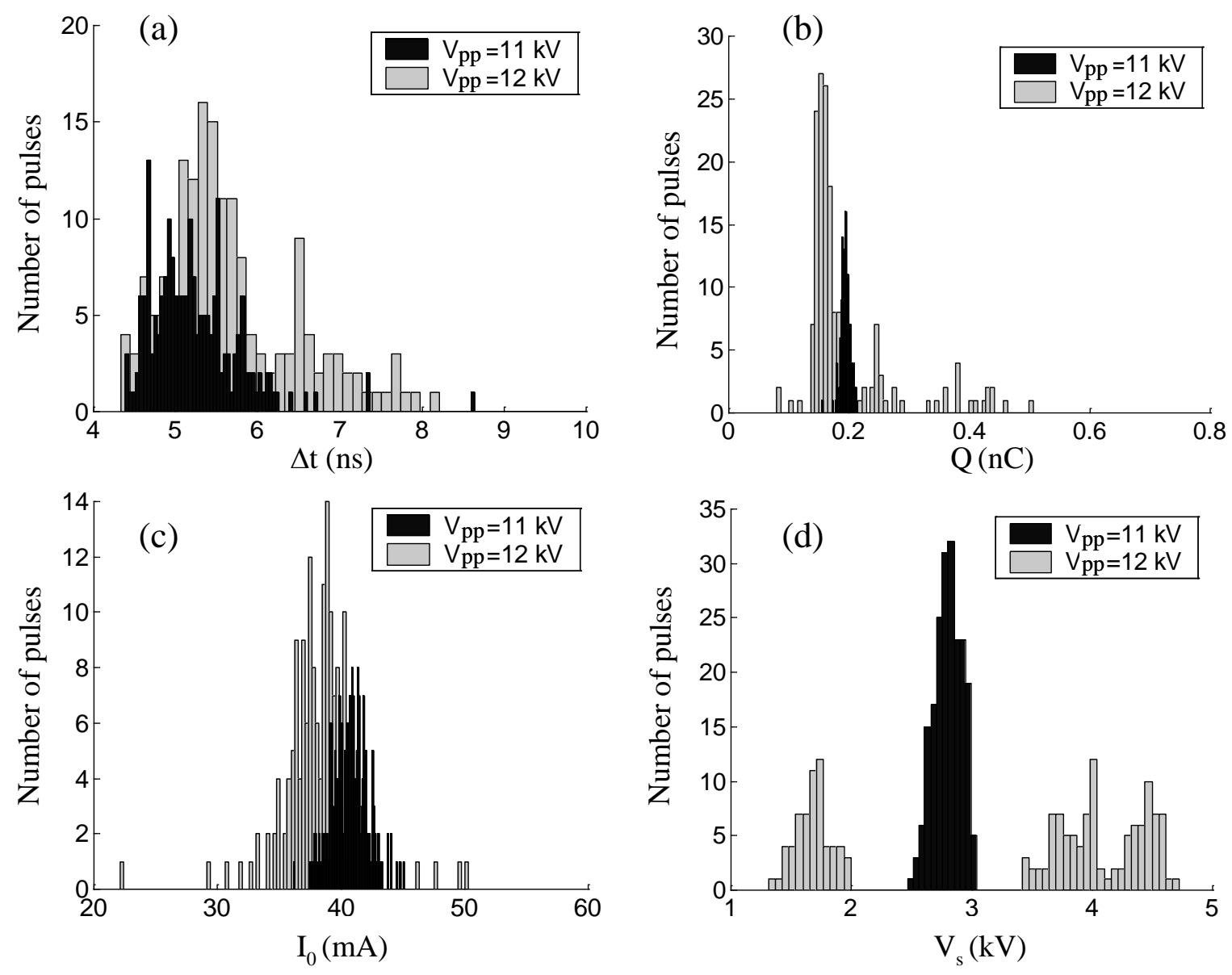

Figure 6: Distributions of the characteristics of the microdischarge in the wire-to-wire arrangement for peak to peak voltages (Vpp) of 11 and $12 \mathrm{kV}$ at $60 \mathrm{kHz}$ (a) Duration (b) Charge (c) Maximum current of the pulse

(d) Instantaneous value of the applied voltage when the pulse occur (Vs)

At $11 \mathrm{kV}$, except for the duration $(\Delta T)$ where current oscillations can disturb the evaluation, all the characteristics present Gaussian distributions. Thus, the average and the standard deviation describe the distributions (cf. Table 1).

At $12 \mathrm{kV}$, some series occur even with only two or three microdischarges per period. The series account for about $30 \%$ of the total number of detected pulses. The dominant mode corresponds to independent microdischarges. The charge and the maximum current of the pulse are linked (cf. discussion below); from 11 to $12 \mathrm{kV}$, they both decrease. The three different Vs correspond to the succeeding microdischarges per half period.

- Identification of the series: definition of the method to study individual microdischarges

In order to define a method to study individual microdischarges, the distribution of charge is plotted for the alumina plane-to-plane arrangement (gap width $1 \mathrm{~mm}$ ) for a peak to peak voltage of $12.8 \mathrm{kV} \mathrm{at} 60 \mathrm{kHz}$ in Figure 7. The weak voltage allows the measurement on the entire plane. The number of filaments per half period is about 20. The plane-to-plane offers a wider surface with more imperfections than the wireto-wire. 


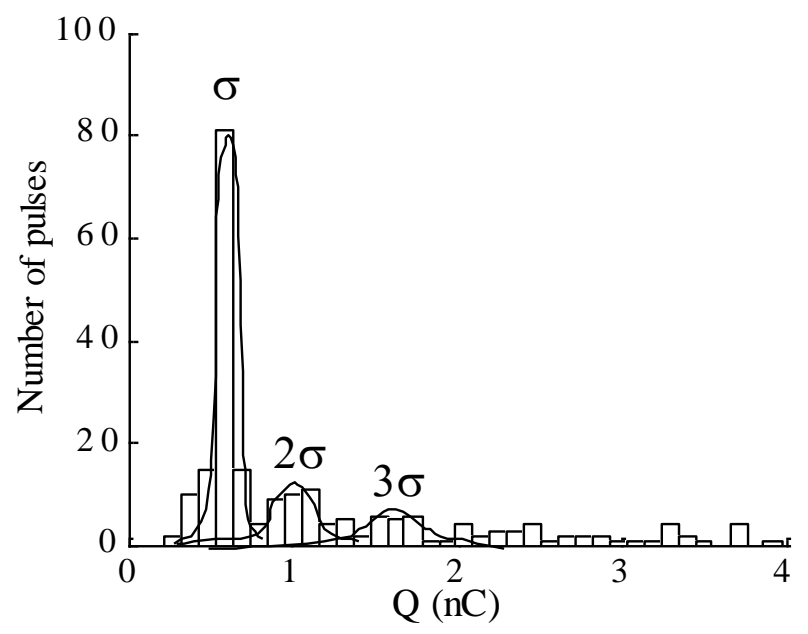

Figure 7: Corrected charge distribution in the alumina reactor.

The dominant mode is centred at $0.55 \mathrm{nC}$. The second mode is around 1.1 and the third one around 1.6. Second and third modes correspond to series with two and three microdischarge. This is confirmed by the standard deviations of the peaks that are proportional to the number of microdischarges per series as already described by Drimal et al. [12]. Series thus correspond to similar microdischarges occurring at the same instant on different locations of the plane electrode.

The dominant mode represents separated microdischarges. In this case, the first mode can be separated; the charge distribution is Gaussian with an average of $450 \mathrm{pC}$ a standard deviation of about $53 \mathrm{pC}$. The smallest charge amount measured for a single microdischarge is about $360 \mathrm{pC}$ whereas the highest is about $950 \mathrm{pC}$. Moreover the charge and the current amplitude of the microdischarge are proportional which helps to exclude the series.

As microdischarges involved in the series have the same characteristics as individual microdischarges, the statistical treatment can focus on individual microdischarges. In order to separate the series microdischarges, the data of the wire-to-wire arrangement presented on the Figure 6 are plotted in the $Q-I_{0}$ plane for both voltages in Figure 8 (a). This representation shows the relation between these two characteristics and allows the distinction between single microdischarge characteristics (general trend) and series in the frame (two or three time higher than the main trend). The data for the plane-to-plane presented in Figure 7 are plotted in Figure 8 (b).

(a)

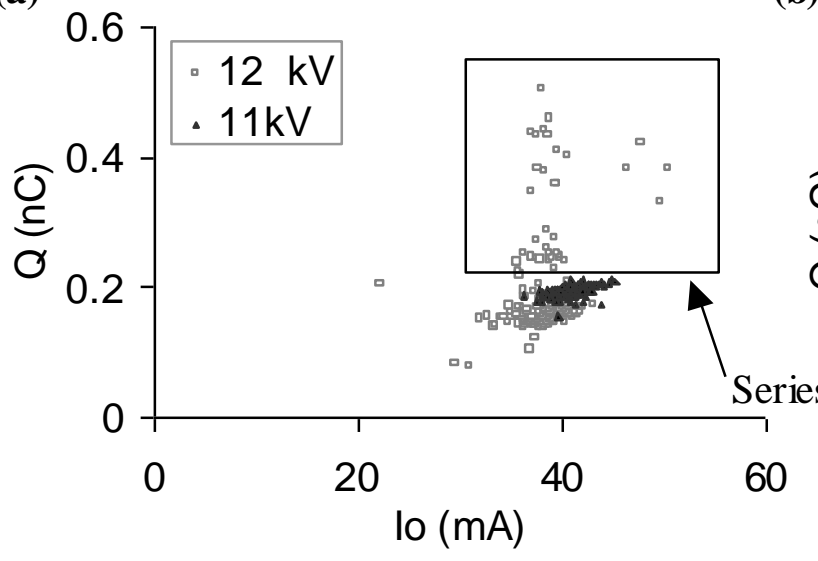

(b)

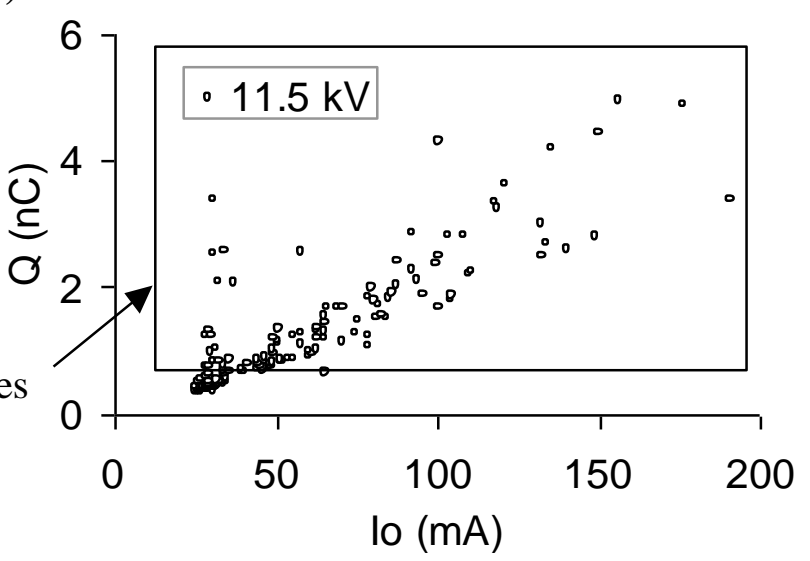

Figure 8: Representation of the pulses in the $Q-I_{0}$ plane for (a) wire-to-wire and (b) plane-to-plane arrangements. 
For individual microdischarges, $Q$ and $I_{0}$ are proportional. At $11 \mathrm{kV}$ (in black in Figure 8), all the dots are regrouped. But at $12 \mathrm{kV}$, the dots corresponding to series of microdischarges are distinct from individual microdischarges points. In this case, individual microdischarges can be separated and analyzed with the statistic data treatment. As expected, with bigger electrode surface, for plane-to-plane, in Figure 8 (b), series are more frequent. With this method series can now be excluded.

The average characteristics ( $I_{0}$ and $Q$ are now corrected with the value of the equation (3), the factor $\alpha$ is 1.4 for the wire-to-wire) and the standard deviations are given in the Table 1 for two voltages.

Table 1: Corrected characteristics of the microdischarge in the wire-to-wire arrangement.

\begin{tabular}{lllll}
\hline \multirow{2}{*}{ Characteristic } & \multicolumn{2}{c}{ Averages } & \multicolumn{2}{c}{ Standard deviation } \\
\cline { 2 - 5 } & $11 \mathrm{kV}$ & $12 \mathrm{kV}$ & $11 \mathrm{kV}$ & $12 \mathrm{kV}$ \\
\hline$\Delta t(\mathrm{~ns})$ & 5.2 & 5.7 & 0.56 & 1.2 \\
\hline$Q(\mathrm{pC})$ & 270 & 220 & 10 & 27 \\
\hline$I_{0}(\mathrm{~mA})$ & 57 & 53 & 2.1 & 3.8 \\
\hline$V_{s}(\mathrm{kV})$ & 2.8 & 3.10 & 0.13 & - \\
\hline
\end{tabular}

From 11 to $12 \mathrm{kV}$, the characteristics vary by about $15 \%$. The voltage increase implies the charge reduction related to the reduction of the maximum current in spite of an increase of the duration. With the voltage increase, some discharges occur on new sites requiring higher applied voltage to reach the breakdown field strength and initiate microdischarge. Thus, the average of $V_{s}$ increases and the standard deviations are multiplied by two.

\subsubsection{Voltage}

Once the protocol of the series exclusion established, the influence of the voltage on a wider voltage range is studied in wire-to-wire and plane-to-plane alumina arrangements. The evolutions of the average of the four characteristics are plotted in Figure 9 for the wire-to-wire arrangement. The first two points concern only one microdischarge per half period as already discussed in section 3.1.2) Then the number of microdischarges increases as described in the Figure 4 (b).
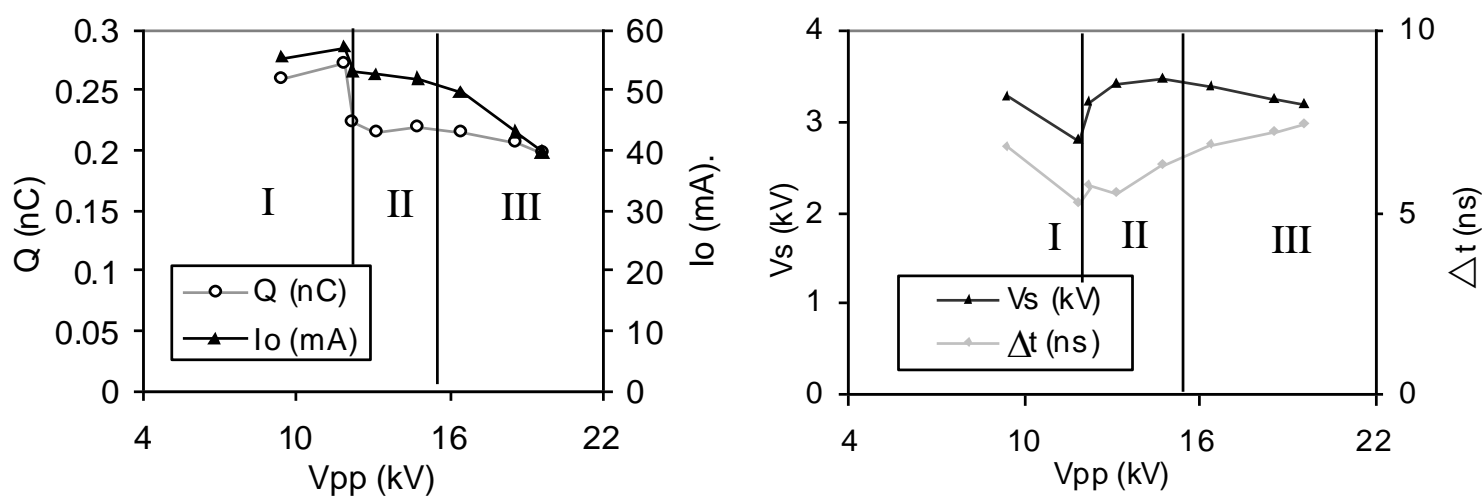

Figure 9: Evolutions of the averages characteristics with the voltage in the wire-to-wire arrangement. 
The three zones identified with macroscopic currents can also be differentiated through the averages of electrical characteristics of the microdischarge.

In zone II, the average characteristics are constant because of the retro control of the electric field due to charges deposited on the surface from one half period to another. The charges deposited on the dielectric by a microdischarge only affect the development of the next microdischarge at the same location in the next half period. There is no interaction between microdischarges during the same half period because they develop further than the influencing volume.

In zone III, $Q, I_{0}$ and $V_{s}$ decrease with the applied voltage. The amounts of charge of surrounding microdischarges, during the same half period, influence the development of each a microdischarge, during the same half period. The reduction of the distance between microdischarges of the same half period increases the electrostatic influence and leads to a shielding effect of surface charges.

The transition between zone II and III allows the evaluation of the distance of influence of the charge deposited on the dielectric by microdischarges. At this point, 8 microdischarges occur on $1 \mathrm{~cm}$. The distance of influence on alumina is about $1.2 \mathrm{~mm}$, which is the same order of magnitude than calculated by Gibalov [1].

The evolutions of the standard deviations are not presented but they all increase with the voltage.

- Plane-to-plane

For low voltage, the identification of the dominant mode corresponding to unitary microdischarge (the highest peak of the distribution) is easy. However, for higher voltage, as the deviation increases, the first and the second modes merged. The identification of a single microdischarge is difficult and nearly impossible for the highest voltage (a solution would consist of adapting the size of the electrode with the voltage). The dominant mode allows the evaluation of the average characteristics. Q and $I_{0}$ are plotted versus peak-to-peak voltage in Figure 10 (a). Figure 10 (b) represents the number of detected pulses including series (dotted line) and the total number of microdischarges (black line).

(a)

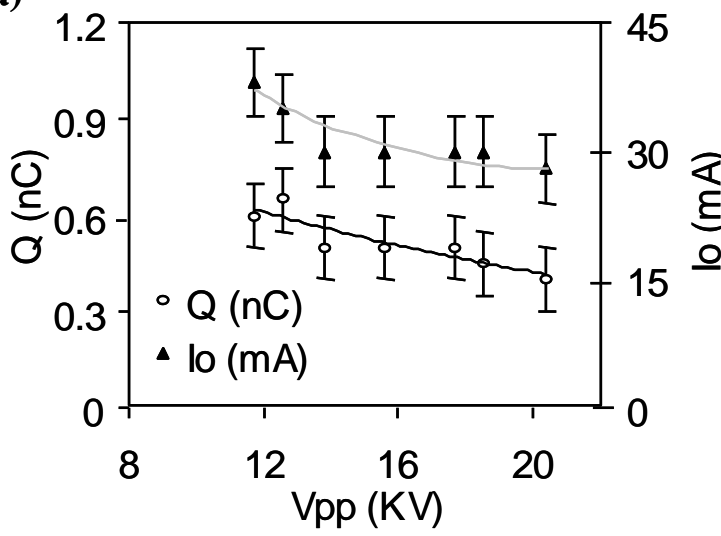

(b)

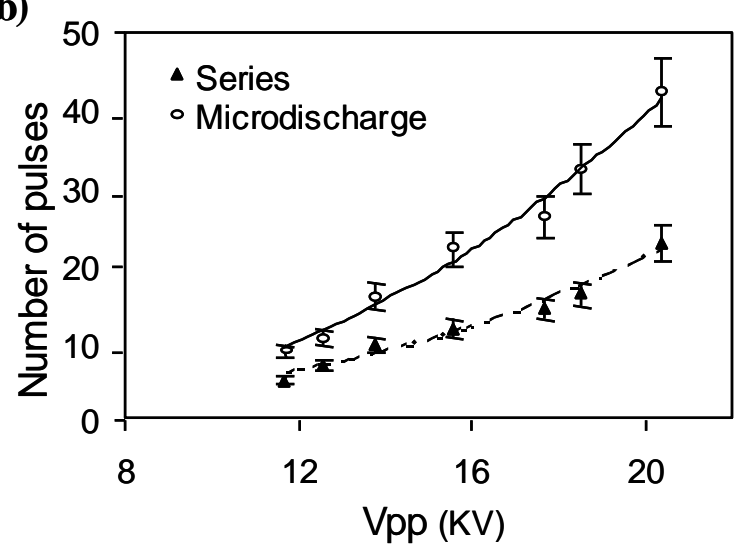

Figure 10: Evolution of the charge and the maximum current with the voltage in the alumina reactor (a) and series and microdischarge number (b).

The alumina reactor operates in mode II, but contrary to the wire-to-wire arrangement, the average characteristics of the microdischarge are not constant. $Q$ and $I_{0}$ decrease with the voltage. The average charge evolves by less than $25 \%$. The surface imperfections and the modification of the gas properties (temperature -cf. section 3.2.4- and composition) all along the reactor can be involved.

In the wire-to-wire arrangement, the distance of influence is $1.2 \mathrm{~mm}$ (cf. section 3.1.2) corresponding to 40 microdischarges per half period per $\mathrm{cm}^{2}$ (obtained at $20 \mathrm{kV}$ ). 


\subsubsection{Gas flow rate}

The influence of the gas flow rate on the microdischarges characteristic is established with the wire-towire arrangement. Only one microdischarge occurs per half period. The $Q-I_{0}$ plane is represented for two gas flow rates $\left(0.2\right.$ and $\left.4 \mathrm{~L} \mathrm{~min}^{-1}\right)$ in Figure 11 (a). The evolutions of the four characteristics with the gas flow rate are plotted in Figure 11 (b).

(a)

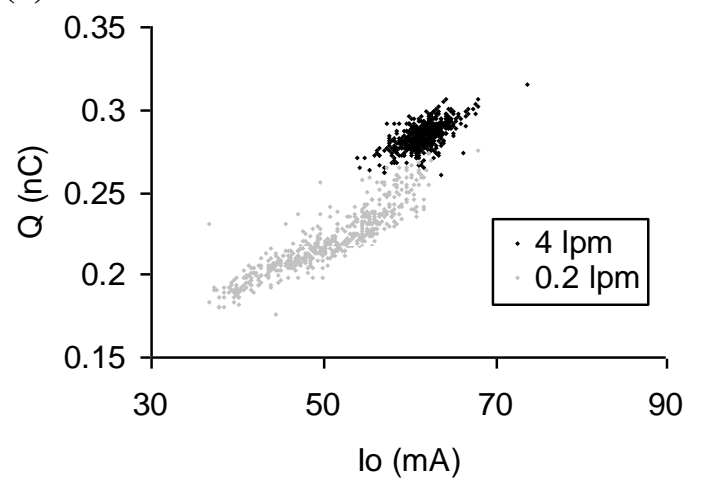

(b)

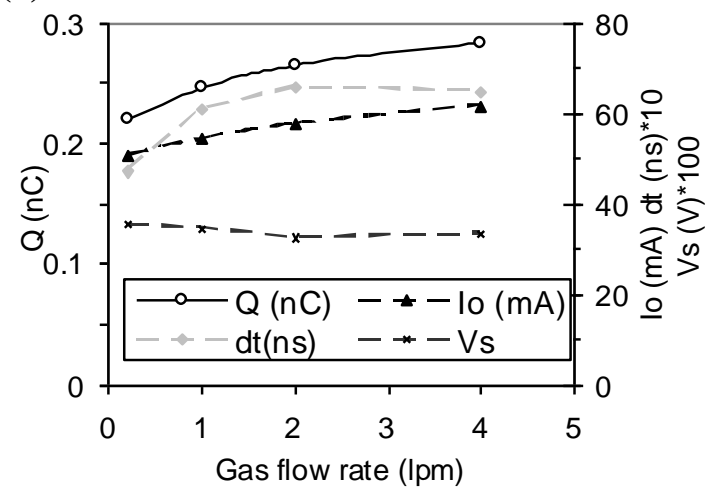

Figure 11: (a) Charge $(Q)$ versus the maximum of the pulse current $\left(I_{0}\right)$ for each microdischarge with two gas flow rates $\left(0.2\right.$ and $4 \mathrm{~L} \mathrm{~min}^{-1}$ ) (b) Evolution of the characteristics with the gas flow rate.

$\left\langle I_{0}>\right.$ and $\langle Q>$ increase with the gas flow rate and the deviation of the characteristic decreases The mean time between two microdischarges occurring at the same location is the half period $(8.3 \mu \mathrm{s} \mathrm{at} 60 \mathrm{kHz})$, in that time the gas moves of about $55 \mu \mathrm{m}$ at $4 \mathrm{~L} \mathrm{~min}^{-1}$. The average characteristics do not differ by more than $20 \%$. The substitution of a part of the gas in the microdischarge volume with fresh air can explain the stabilization of the discharge with the gas flow rate. The gas flow rate may also increase the extension of the charges on the surface leading to weaker retro control of the charge deposit. When charges are spread on the surface, the electric field is higher during the development of the next discharge leading to higher maximum current and charge of the following pulse. With gas flow rate below $0.2 \mathrm{~L} \mathrm{~min}^{-1}$, the microdischarges can't be considered identical, as the deviations of the characteristics are too important.

\subsubsection{Temperature}

For a constant voltage of $15 \mathrm{kV}$ at $60 \mathrm{kHz}$ with the cordierite reactor and a gas flow rate of $2 \mathrm{~L} \mathrm{~min}^{-1}$, the output temperature of the gas evolves from ambient temperature to $65^{\circ} \mathrm{C}$. In the same time, the number of microdischarges increases by about $10 \%$ with the temperature and both maximum current and duration decrease leading to a diminution of $\langle Q>$ of about $15 \%$. The mean characteristics at ambient temperature and for temperatures above $100^{\circ} \mathrm{C}$ differ more than $20 \%$, the microdischarges can't be considered identical anymore.

At least two parameters are affected by the temperature: the gas density and the surface conductivity of the dielectric material. The reduction of the gas density leads to a reduced breakdown voltage i.e. a higher number of microdischarges will appear. The increase of the surface conductivity modifies the distribution of the charges on dielectric surfaces, the electric field in the microdischarge and thus the related characteristics. Indeed, the influence of the temperature can also explain the reduction of the average charge with the voltage as observed in section 3.2.2.

It has to be underlined that the surface temperature is higher than gas temperature; thus the gas is heated by hotter surfaces [26]. In given conditions, i.e. with similar microdischarges, the number of microdischarge per surface and times units defines the final surface temperature. One has to keep mind that this coupling of electrical and thermal characteristics can be modified by tuning either the thermal capacitance of the reactor or the thermal exchanges around the reactor. This enables to prove that, in millimetre plane-to-plane arrangements, the temperature of the electrode is the critical parameter to control the nature of the chemical species produced in dry air. Indeed for similar electrical characteristic of 
microdischarge ozone is dominant for electrode temperature below $150{ }^{\circ} \mathrm{C}$ whereas the nitrogen oxides are dominant above $150{ }^{\circ} \mathrm{C}[26]$.

\subsubsection{Gap width}

This statistical study of the microdischarge characteristics confirms the influence of the gap width, already described in many other studies. The average charge is proportional to the gap width. The average charge at $0.5 \mathrm{~mm}$ is about $0.22 \mathrm{nC}$ and $0.5 \mathrm{nC}$ at $1 \mathrm{~mm}$. Both the maximum and the duration of the microdischarge increase with the gap width.

\subsubsection{Frequency}

The influence of the frequency is established in the $0.5 \mathrm{~mm}$ gap alumina reactor. The comparisons of the characteristics of the microdischarge at $1 \mathrm{kHz}$ and $60 \mathrm{kHz}$ for the same voltage $8.6 \mathrm{kV}$ are plotted in Figure 12.
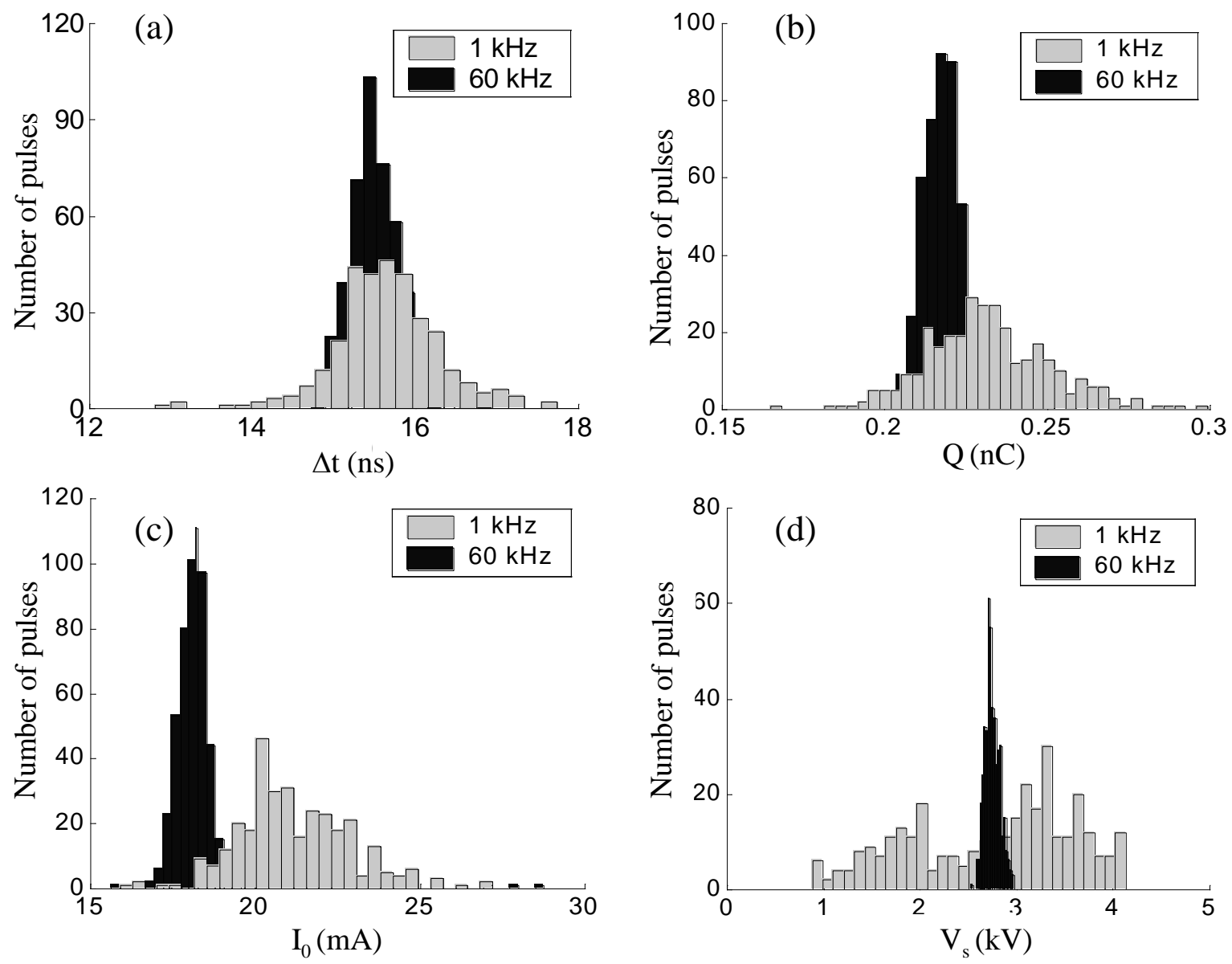

Figure 12: Distribution of the characteristics of the microdischarges in plane-to-plane arrangement at 1 and $60 \mathrm{kHz}$ for a $0,5 \mathrm{~mm}$ gap (a) Duration (b) Charge (c) Maximum current (d) Instantaneous value of the applied voltage when the pulse occur $(V s)$.

The duration is independent of the frequency. The maximum current, the charge and their standard deviations increase with the reduction of the frequency. The range of the instantaneous value of the applied voltage $\left(V_{s}\right)$ is only of $0.5 \mathrm{kV}$ at $60 \mathrm{kHz}$ whereas it reaches $3.3 \mathrm{kV}$ at $1 \mathrm{kHz}$.

The increase of the average charge is even more noticeable at $1 \mathrm{~mm}$ (up to $40 \%$ ) and can be induced by the charge migration on the surface. The time between two microdischarges at the same location increase 
with the reduction of frequency. Charges deposited on surface have more time between two successive half periods to spread on the surface reducing their effect on the field in the gas.

The reduction of the half period duration can explain the difference between the numbers of microdischarge per half period at 1 and $60 \mathrm{kHz}$. As the voltage is low, near the voltage of extinction, the probability of initiation of a microdischarge is highly dependant on the time lag with a high electric field in the gas.

\subsubsection{Comparison between plane-to-plane arrangements with or without walls in the gaps}

Alumina with a $1 \mathrm{~mm}$ gap and cordierite cells have similar specific capacitances ( $\varepsilon / \mathrm{e})$. However the microdischarges possess a different shape. The average charges per microdischarge are approximately 0.2 $\mathrm{nC}$ for cordierite compared to $0.5 \mathrm{nC}$ for alumina. The total duration of the microdischarge is much smaller for cordierite (5 ns against $30 \mathrm{~ns}$ for alumina). In the cordierite reactor, the wall of the ceramic may lead to faster propagation of microdischarges by electron emission from the surface. Photo-ionization in the gas region requires UV photons of more than $10 \mathrm{eV}$. Photo-emission from surfaces requires photon energies of only 4-6 eV [27]. The more rapid pulse decrease is linked to the electron attachment, because of the surface polarization and the resulting reduction of the electric field in the gas when discharge propagates on a surface.

However, for similar energy density with different electrical characteristics in both cells, ozone concentrations differ less than $10 \%$ of. Thus the interest of this arrangement for pollution control lies on enlarged surface to volume ratio involved in heterogeneous catalytic plasma processes rather than on the modification of electrical characteristics affecting primary species.

\subsubsection{Charge density on the ceramic}

The accumulation and displacement of charge on the dielectric surface is essential for the characterization of the DBD. The charge of a microdischarge differs from the charge remaining on the surface after the development of the microdischarge. This is because, on the one hand, part of the charge is used to neutralize the preceding charges deposited on the surface, and on the other hand, not all the charges moving in the gap are collected on the surfaces. Some charges could be neutralized in the volume and some can remain in the gap once surfaces are polarized.

Moreover, the volume of influence may be of particular importance when dealing with the charging of solid particles injected in plane-to-plane reactors [28]. In fact, efficient charging conditions (when the average product of the ion density by the time in this ion density "seen" by the particle is upper than $10^{7}$ $\mathrm{s.cm}^{-3}$ ) are encountered when particle passes through this influence volume of a microdischarge.

The two first half periods succeeding the first switch on of the wire-to-wire can illustrate the influence of the charge on surface. The very first microdischarge $(1.1 \mathrm{nC})$ occurs at maximum voltage $7 \mathrm{kV}(5 \mathrm{kV} / \mathrm{mm}$ in the gap). The first microdischarge of the second half period $(0.8 \mathrm{nC})$ occurs at zero voltage. Assuming that these two microdischarges occur at the same location because of the electric field reinforcement above the charge deposited on the surface, the charge of $1.1 \mathrm{nC}$ induces an electric field of $5 \mathrm{kV} / \mathrm{mm}$ on the axis of microdischarge. The charge density on the surface is about $\sigma=8 \mathrm{nC} \mathrm{cm}^{-2}(E=\sigma / \varepsilon)$ at the feet of the first microdischarge and the charges spread on a disc of $2 \mathrm{~mm}$ diameter.

When only one discharge occurs in the wire-to-wire cell, the average charge is $0.2 \mathrm{nC}$ and the pulse voltage is $2.8 \mathrm{kV}$ (cf. Figure 6). The charges induce an electric field of $3 \mathrm{kV} / \mathrm{mm}$ on the axis of microdischarge. The charge density is about $4.8 \mathrm{nC} \mathrm{cm}^{-2}$ and the diameter is about $0.6 \mathrm{~mm}$ by assuming half of the charge $(0.1 \mathrm{nC})$ is deposed on the surface. Moreover, the volume of influence of the microdischarge is evaluated as a cylinder with a circular base of $1.2 \mathrm{~mm}$ diameter (twice the area of the charge deposit).

With alumina with a specific capacitance of the dielectrics (including the silicon paste) of $1.5 \times 10^{-8} \mathrm{~F} \mathrm{~m}^{-2}$ and a gap width of $1 \mathrm{~mm}$, the volume of influence is about $1.1 \mathrm{~mm}^{3}$. The number of microdischarges that can develop during the same half period without interaction between microdischarges is about 80 per $\mathrm{cm}^{2}$. 



\section{Conclusions}

A method has been developed to achieve representative statistical distribution of electrical characteristics of the microdischarges, by excluding the microdischarge series in millimetre sized plane-to-plane dielectric barrier discharge with sinusoidal voltage. The relative influence of the operating parameters (voltage, gas flow rate, gap width, frequency) on the local surface polarization, electron attachment and temperature affecting microdischarge characteristics has been depicted in different arrangements.

With similar specific capacitance, the influence of the gap width on the electrical characteristics of plane-to-plane arrangement has been confirmed.

In given conditions, i.e. with similar microdischarges, the number of microdischarge per surface and time units defines the final surface temperature. One has to keep mind that this coupling of electrical and thermal characteristics can be modified by tuning either the thermal capacitance of the reactor or the thermal exchanges around the reactor.

Higher gas temperatures increase the number of microdischarge per surface and time units due to the reduction of the gas density and reduce the average charge per pulse.

This method enables to prove that, in millimetre plane-to-plane arrangements, the temperature of the electrode is the critical parameter to control the nature of the chemical species produced in dry air. Indeed for similar electrical characteristic of microdischarge ozone is dominant for electrode temperature below $150{ }^{\circ} \mathrm{C}$ whereas the nitrogen oxides are dominant above $150{ }^{\circ} \mathrm{C}$.

This method is also used to depict, for the first time, the influence of dielectric walls perpendicular to the electrodes inside the discharge gap on microdischarge electrical characteristics in the case of the single stage cordierite monolith widely used in pollution control reactors. Indeed, a faster pulse decrease due to an enhanced electron attachment is reported when discharge propagates on a surface leading to less than $10 \%$ of discrepancy on ozone concentration for similar energy density. Thus the interest of this arrangement for pollution control lies on enlarged surface to volume ratio involved in heterogeneous catalytic plasma processes rather than on the modification of electrical characteristics affecting primary species.

Besides, this study allows the identification of the operating conditions for which all the microdischarges can be considered identical to study local physical and chemical processes around each microdischarge. For given operating conditions, the microdischarges can be considered identical if the deviation of each electrical characteristic of the pulses is lower than $\pm 30 \%$ of the average. In a given reactor, the microdischarges can be considered identical if the average electrical characteristics of the pulses do not vary more than $20 \%$ with the variation of one operating parameter. This experimental study shows that to keep similar microdischarges, the gas temperature and the flow rate have to lie within defined ranges.

With similar microdischarges, the distinction between spatial and temporal interaction of microdischarges can thus be envisaged from macroscopic electrical measurements. Indeed, the applied voltage controls the number of microdischarge per period and the frequency of the voltage controls the repetition of the microdischarges at the same location. This enables to work with defined number of microdischarge "seen" by an elementary unit volume of gas transiting in the gap, so that local physical and chemical processes around each microdischarge can be studied from macroscopic concentration measurement in the exhaust in different coupling conditions of similar microdischarges.

This could be done, for instance, for ozone production, pollution control reactors and even for the mechanisms involved in the production of solid particles by plasma. Indeed, the chemical modelling of a microdischarge is accurate to account for the output concentrations of chemical species at low repetition rate per surface units, until the vicinity of microdischarge affects the density and thermal gradients between microdischarges. In that case, the evaluation of the number of similar microdischarge allows the extrapolation of microdischarge models to the whole reactor by defining an empirical function depending on the operating parameters to account for the electro-thermal coupling which affects the transport and the kinetics of similar primary species. 


\section{Acknowledgement}

Thanks to Doctor Kirill V. Kozlov for useful discussions.

\section{References}

[1] Gibalov V I and Gerhard J Pietsch 2000 The development of dielectric barrier discharges in gas gaps and on surfaces J. Phys. D: Appl. Phys 33 2618-36

[2] Kozlov K V, Wagner H-E, Brandenburg R and Michel P 2001 Spatio-temporally resolved spectrometric diagnostics of the barrier discharge in air at atmospheric pressure J. Phys. D: Appl. Phys 34, No 11, $3164-76$

[3] Eliasson B, Hirth M, Kogelschatz U 1987 Ozone synthesis from oxygen in dielectric barrier discharges $J$ Phys. D: Appl. Phys 20 1421-37

[4] Kogelschatz U, Eliasson B and Egli W 1999 From ozone generators to flat television screens: history and future potential of dielectric-barrier discharges Pure Appl. Chem., Vol 71 , No 10, 1819-28

[5] Fridman A, Chirokov A and Gustol A 2005 Non-thermal atmospheric pressure discharges J. Phys. D: Appl. Phys. 38 R1-R24

[6] Hammer T 1999 Application of plasma technology in environmental techniques Contrib. Plasma Phys. 39 44162

[7] McAdams R 2001 Prospects for non-thermal atmospheric plasmas for pollution abatement J. Phys. D. : Appl. Phys, 34 2810-21

[8] Bogaerts A, Neyts E, Gijbels, Van Der Mullen J. 2002 Gas discharge plasmas and their applications Spectrochimica Acta Part B57 609-58

[9] Xu X. P. and Kushner M J 1998 Ion composition of expanding microdischarges in dielectric barrier discharges J. Appl. Phys 83 N $127522-32$

[10] Marode E et al. 1997 Influence of temperature, hydrodynamic and diffusion process on chemical activity in filamentary discharge INSTP 2nd inter. Symp on non thermal plasma technology for gaseous pollution control, Salvador, Bahia Brasil 130-35

[11] Kreuger F H and Gulski E 1989 Automatisiertes Mehlsystem zur Erfassung von Teilentladungs-Kenngrossen für die Beurteilung elektrischer Isolierungen” Tech. Mess. 56 124-9

[12] Drimal J, Gibalov V and Samoilovich V 1987 Silent discharge in air, nitrogen and argon Czech. J. Phys. B 37 1248-55

[13] Gibalov V I, Novikov P S, Rakhimov A T,.Saenko V B and Saveliev A B 2000 The structure and productivity of the barrier discharge Proc. Of XIII Int.Conf. on Gas Disch. and their Appl. Glasgow, 291-4

[14] Sato N 1980 Discharge current induced by the motion of charged particles J. Phys. D. : Appl. Phys 13 L3-6

[15] Morrow R and Sato N 1999 The discharge current induced by the motion of charged particles in time dependent electric fields; Sato's equation extended J. Phys. D: Appl. Phys 32 L20-2

[16] Raether H. 1964 Electron avalanche and breakdown in gases (London: Butterworths)

[17] Drimal J, Kozlov K V, Gibalov V and Samoilovich V 1988 On value of transferred charge in silent discharge under atmospheric pressure Czech. J. Phys. B 38 159-65

[18] Falkenstein Z and Coogan J J 1997 Microdischarge behaviour in the silent discharge of nitrogen - oxygen and water - air mixtures J. Phys. D: Appl. Phys. 30 No 5 817-25

[19] Petit M, Jidenko N, Goldman A, Goldman M and Borra J P 2002 Electrical characterization of gas discharges using a numerical treatment. Application to dielectric barrier Discharges Rev. Sci. Instrum. 73 No7 2705-12

[20] Bibinov N.K., Fateev A A and Wiesemann 2001 On the influence of metastable reactions on rotational temperatures in dielectric barrier discharges in He- $\mathrm{N}_{2}$ mixtures particles J. Phys. D.: Appl. Phys 34 1819-26

[21] Bagirov M A, Nualiev H A and Kurbanov M A 1972 Investigations on a discharge in an air gap limited by a dielectric and method of determining the number of partial discharges J. Tech. Phys. 43 629-34 (in Russian)

[22] Murata T, Tatsukawa M., Okita Y. and Yasuoka K. 1995 Polarity Effect of Silent Discharge Ozone science \& engineering Vol 1757

[23] Petit M, Goldman A and Goldman M 2002 Glow currents in a point-to-plane dielectric barrier discharge in the context of the chemical reactivity control J. Phys. D: Appl. Phys. 35 No 22 2969-77

[24] Jidenko N and, Petit M, Borra J P 2002 Volatile organic compounds depollution by dielectric barrier discharge in porous media (Contributed papers Int. Symp. On high Pressure Low Temp. Plasma Chem. (Hakone VIII)) 364-8

[25] Parissi L, Goldman A, Goldman M, Odic E, Borra J P 2000 Electrical Discharges for Environmental Purposes, E van Veldhuizen ed, Raizers Book, New York, chapter 11 279-312 
[26] Jidenko N 2004 Caractérisation électrique et couplages électro-thermiques des décharges à barrières diélectriques dans l'air à pression atmosphérique: Faisabilité de l'électrofiltration d'aérosol $P h D$ Thesis University of Paris XI Orsay

[27] Gibalov V I and Gerhard J Pietsch 2004 Dynamics of dielectric barrier discharges in coplanar arrangements $J$. Phys. D: Appl. Phys. 37 2082-92

[28] Borra J-P 2006 Nucleation and aerosol processing in atmospheric pressure electrical discharges: powders production, coatings and filtration J. Phys. D: Appl. Phys. To be published 